\title{
Coronavirus disease 2019 in a 13-year-old patient with acute lymphoblastic leukemia
}

On March 2, 2020, a 13-year-old female patient has been admitted to Hazrat-e Fateme Masoume Hospital, Qom city, Iran with the main complaint of pallor, anemia and weight loss. Bone marrow smears show hypercellular bone marrow aspiration composed of more 50\% blastic cells with high nucleus/ cytoplasm ratio, fine chromatic, 2-3 prominent nuclei, and thin rim of basophilic cytoplasm. Ultimately, flowcytometry assay was confirmed acute lymphoblastic leukemia (ALL) with T-cell type. The patient was reported to have high-risk ALL. The patient underwent medication with chemotherapy agents in Table 1. On March 12, 2020, once again, the patient has been admitted to the hospital with the main complaint of fever $\left(38.5^{\circ} \mathrm{C}\right)$ and vomiting. Also, the patient presented gingival bleeding, cough, and myalgia. Laboratory test for severe acute respiratory syndrome coronavirus 2 (SARS-CoV-2) was performed using real-time reversetranscriptase polymerase chain reaction (rRT-PCR) that was reported positive. The cycle threshold $(\mathrm{Ct})$ value was reported
25. Complementary evaluation was followed by performing chest computed tomography (CT) scan which showed a welldefined nodular ground-glass opacity with diameter of $6 \mathrm{~mm}$ at lateral segment of RML (Fig. 1A, B). Despite lung involvement, gasometer data were in normal range. Within 2 weeks, all symptoms for SARS-CoV-2 were subsided. Laboratory rRTPCR test was repeated that was reported negative $(\mathrm{Ct}$ value $=39)$. Additionally, radiologic findings disappeared. On April 18, 2020, the patient has been admitted to hospital and received treatment with chemotherapy agents for third time. Three days later, the patient has been hospitalized again with the main complaint of fever $\left(38.7^{\circ} \mathrm{C}\right)$, abdominal pain, headache, diarrhea, and nausea, as at the beginning of hospitalization, she presented tonic-clonic seizures twice and lost consciousness as well. Drowsiness and stiff neck were evident in the patient. Chest CT scan was requested once again as revealed small bilateral peripheral consolidations with ground-glass opacity and a crazy paving pattern $(\mathrm{Ct}$ value $=$
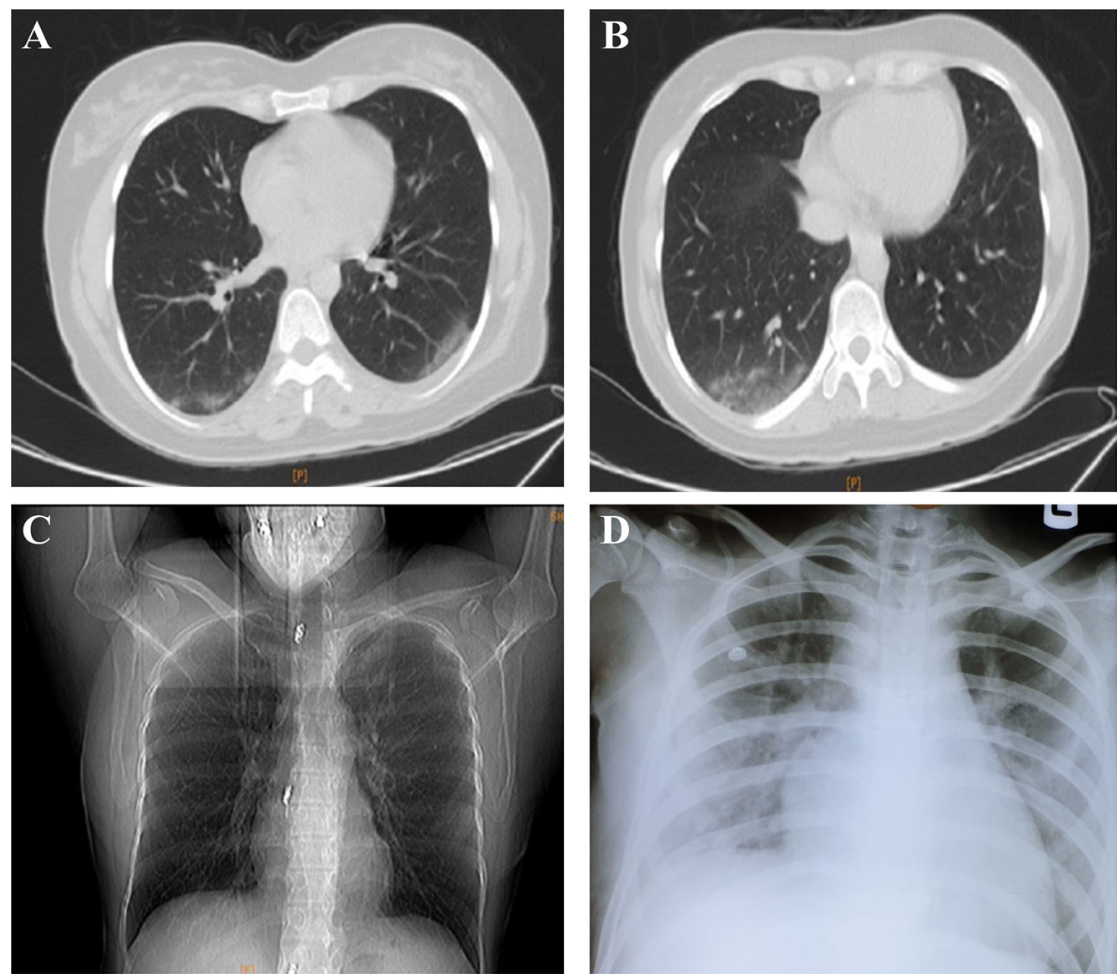

Fig. 1. Chest computed tomography scan (transverse plane) of the 13-year-old female patient revealing nodular ground-glass opacity and white lung on the last day of hospitalization. (A, B) Chest computed tomography scan (transverse plane) of the 13-year-old female patient revealing nodular ground-glass opacity and (C, D) white lung on the last day of hospitalization. 
27 in rRT-PCR). The patient underwent medication, but eventually, the patient's lung turned white (Fig. 1C, D) and died due to the acute respiratory distress syndrome and cardiovascular arrest.

\section{COVID-19 and ALL}

The novel coronavirus disease 2019 (COVID-19) is affecting the entire population of the world after its outbreak in Wuhan, China. ${ }^{1)}$ It has been well documented that definitive diagnosis of COVID-19 in children is less than 2\%. Relatively few pediatric COVID-19 cases were hospitalized, as mainly limited to the pediatric patients aged $<1$ year and those with underlying conditions. ${ }^{2,3)}$ ALL numerate as the most common form of childhood malignancy that originates in a single B- or T-lymphocyte progenitor. It is reported that the account for the peak incidence is in early childhood. ${ }^{4}$ ) So far, most of the published data on COVID-19 infection is mainly focused on children and newborns ( $<18$ years) and there is little information about infected children with cancer, particularly ALL. ${ }^{4)}$

\section{Treatment of ALL in COVID-19}

There have not yet been any international guidelines developed to address the optimal management of ALL patients in any infectious pandemic. ${ }^{5)}$ Therefore, at the age of COVID-19, it seems that the disease management in children with ALL is sometimes challenging and of high importance. The novel COVID-19 outbreak is rapidly affecting children with cancer, and it seems that special care is required for these patients. Regarding various case reports have described the impact of COVID-19 in the pediatric population, the impact of COVID-19 pneumonia on children with cancer need more investigation. ${ }^{4,6)}$ So far, the medical management of children with cancer, remains unclear and poorly documented.

In a study, the clinical course of COVID-19 in childhood with acute leukemia was reported by Chen et al. ${ }^{6}$ The patient was reported to have high-risk ALL who on his maintenance chemotherapy neutropenic fever and cough had occurred. The child remained febrile and the requested chest CT scan for the second time 11 days after symptom onset showed progressive changes. The patient then was isolated and treated with umifenovir, ribavirin, and recombinant interferon $\alpha-1 b$ nebulized inhalations. Seven days later, the child's blood count had recovered and the SARS-CoV-2 test result became negative; however, four days later, the test was reported positive again and he was transferred to the intensive care unit.

In this case report, we documented one COVID-19 patient with presentation of ALL. The patient underwent chemotherapy and was discharged from the hospital. After chemotherapy, the patient presented symptoms of COVID-19 infection. The results of clinical evaluation, rRT-PCR tests, as well as Ct values, confirmed the reinfection or reactivation with the SARS-CoV-2.

Table 1. Laboratory examination results of a 13-year-old patient who had coronavirus disease 2019 and acute lymphoblastic leukemia

\begin{tabular}{|c|c|c|c|c|}
\hline Variable & March 2 & March 12 & April 21 & Reference range \\
\hline \multicolumn{5}{|l|}{ Test result } \\
\hline LDH (IU/L) & $444^{\mathrm{a})}$ & $900^{\mathrm{a})}$ & $1154^{\mathrm{a})}$ & 10-12 years: $120-293$ \\
\hline Creatinine (mg/dL) & 0.4 & $0.73^{\mathrm{a})}$ & $2.7^{\mathrm{a})}$ & Children: 0.3-0.7 \\
\hline CRP (mg/L) & 3.2 & $39.4^{\mathrm{a})}$ & $60.9^{\mathrm{a})}$ & Quantitative $<6$ : negative \\
\hline Reticulocyte count (\%) & $0.3^{\mathrm{b})}$ & 0.5 & $0.4^{\mathrm{b})}$ & Adults: $0.5 \%-1.5 \%$ \\
\hline \multicolumn{5}{|l|}{ Newborns: 2\%-6\% } \\
\hline $\operatorname{WBC}\left(10^{3} / \mu \mathrm{L}\right)$ & 9,000 & $2,800^{b)}$ & $1,000^{b)}$ & 9-12 years: 4,500-13,500 \\
\hline $\operatorname{RBC}\left(10^{6} / \mu \mathrm{L}\right)$ & $3.86^{\mathrm{b})}$ & $2.56^{\mathrm{b})}$ & $2.86^{\mathrm{b})}$ & 6-12 years: 4.0-5.2 \\
\hline Hemoglobin (g/dL) & $11.1^{\mathrm{b})}$ & $7.2^{b, c)}$ & $7.3^{\mathrm{b})}$ & $12.4-15.7$ \\
\hline Platelet $\left(10^{3} / \mu \mathrm{L}\right)$ & $26,000^{\mathrm{b})}$ & $8,000^{b, c)}$ & $10,000^{\mathrm{b})}$ & $150,000-450,000$ \\
\hline $\mathrm{ESR}(\mathrm{mm})$ & 13 & $88^{\mathrm{a})}$ & 25a) & Children: 3-13 \\
\hline $\mathrm{SpO} 2(\%)$ & 96 & 97.5 & $63.2^{\mathrm{b})}$ & 90-100 \\
\hline PT (sec) & 11 & 13 & $23^{\mathrm{a})}$ & $11-13.5$ \\
\hline $\mathrm{PTT}$ (sec) & 29 & 36 & $58^{\mathrm{a})}$ & $25-45$ \\
\hline D-dimer (ng/mL) & - & - & $18,817^{\mathrm{a})}$ & Up to 500 \\
\hline Troponin I (ng/L) & - & - & 1,188 & T0>100 positive \\
\hline rRT-PCR & Negative & Positive & Positive & \\
\hline Treatment & $\begin{array}{l}\text { Vincristine+doxorubicin+ } \\
\text { prednisolone+PEG Aspar }\end{array}$ & $\begin{array}{l}\text { Hydroxychloroquine+ } \\
\text { lopinavir/ritonavir+oseltamivir+ } \\
\text { vincristine+doxorubicin+ } \\
\text { prednisolone+PEG Aspar }\end{array}$ & $\begin{array}{l}\text { Lopinavir/ritonavir+oseltamivir+ } \\
\text { levetiracetam+methylprednisolone+ } \\
\text { IVIG+platelets+dopamine+FFP+ } \\
\text { pethidine }\end{array}$ & \\
\hline
\end{tabular}

LDH, lactate dehydrogenase; CRP, C-reactive protein; WBC, white blood cell; RBC, red blood cell; ESR, erythrocyte sedimentation rate; PT, prothrombin time; PTT, partial thromboplastin time; rRT-PCR, reverse transcription polymerase chain reaction; TO, troponin I; PEG, PEG is PEG-L-Asparaginase or Oncaspar as anticancer agents; IVIG, intravenous Immunoglobulin; FFP, fresh frozen plasma.

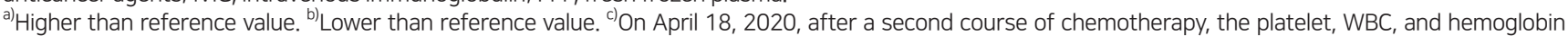
values returned to normal $\left(172,000 \times 10^{3} / \mu \mathrm{L}, 4.9 \times 10^{3} / \mu \mathrm{L}\right.$, and $8.2 \mathrm{~g} / \mathrm{dL}$, respectively). 


\section{How should care the risk of COVID infection in cancer patients?}

Overall, COVID-19 is very fatal for some cancer patients, but most are adults and often have other underlying conditions. Pediatric population is less likely to be infected by COVID-19 as in compared to adults, they may present mild symptoms, though in some cases, especially for patients with cancer, severe infections may occur. All kind of infection including COVID-19 could be the main cause of mortality and morbidity of leukemia patients with chemotherapy. ${ }^{78}$ In this case report, cerebrospinal fluid evaluation, blood culture test, and rRT-PCR were performed to exclude the other reasons of patient deterioration on last admission. With notice to the negative result for blood culture test, various organ involvements including; central nervous system (seizure and loss of consciousness), heart (tachycardia and increasing of troponin), severe increasing of ferritin and D-Dimer, history of positive rRT-PCR, we claim that the major cause of a death may be related to the COVID-19 infection or maybe second complications of COVID-19. Multisystem inflammatory syndrome (MIS-C) in children is a serious condition that appears to be linked to COVID-19. In rare cases, MIS-C could result in permanent damage or even death. It seems that this syndrome was occurred in this patient who was infected with the COVID-19 (according to the WHO diagnostic criteria for MIS- $\mathrm{C}^{9}$ ). The exact cause of MIS-C in children with COVID-19 is not known yet, but it appears to be an excessive immune response related to the SARS-CoV-2. $\left.{ }^{10}\right)$ This case report depicts the importance and fatality of immune suppression after COVID-19 infection, which can cause reactivation or reinfection of the virus. In conclusion, both children and adults with confirmed COVID-19, the patients' medical history can play great role in the incidence of severe symptoms and even death.

\section{Key message}

Question: What should be considered in an immunocompromised child with coronavirus disease 2019 (COVID-19)?

Finding: Due to the importance of appropriately managing COVID-19 in children with cancer, the possibility of a fatal outcome should be considered in immunocompromised patients who receive chemotherapy agents.

Meaning: In all kinds of infections including COVID-19, disuse management and the development of international guidelines for children with cancer is challenging but important.

\section{Conflicts of interest}

No potential conflict of interest relevant to this article was reported.

\section{Ethical statement}

Approval was obtained from the ethics committee of Qom University of Medical Sciences. The procedures used in this study adhere to the tenets of the Declaration of Helsinki (Nu: IR.MUQ.REC.1399.056).
ORCID

Seyed Kamal Eshagh Hosaini (1) https://orcid.org/0000-00020176-4884

Zahra Movahedi $@$ https://orcid.org/0000-0002-5847-6111

Ahmad Hormati $\odot$ https://orcid.org/0000-0002-1322-1318

Hosein Heydari 10 https://orcid.org/0000-0002-8627-9625

Seyed Jalal Eshagh Hosseini 1 h https://orcid.org/0000-00033139-9512

Mahboubeh Afifian @ https://orcid.org/0000-0002-8924-2246

Sajjad Ahmadpour (1) https://orcid.org/0000-0003-4321-874X

Seyed Kamal Eshagh Hossaini, $\mathrm{MD}, \mathrm{PhD}^{1}$, Zahra Movahedi, $\mathrm{MD}, \mathrm{PhD}^{1}$, Ahmad Hormati, MD, $\mathrm{PhD}^{2,3}$, Hosein Heydari, $\mathrm{MD}, \mathrm{PhD}^{1}$, Seyed Jalal Eshagh Hosseini, $\mathrm{MD}, \mathrm{PhD}^{4}$, Fatemeh Khodadust, MSc ${ }^{5}$, Mahboubeh Afifian, $\mathrm{MSc}^{6}$, Sajjad Ahmadpour, $\mathrm{MSc}, \mathrm{PhD}^{3}$

${ }^{1}$ Department of Pediatrics, School of Medicine, Hazrat-e Fateme Masoume Hospital, Qom University of Medical Sciences, Qom, Iran; ${ }^{2}$ Gastrointestinal and Liver Diseases Research Center, Iran University of Medical Sciences, Tehran, Iran; ${ }^{3}$ Gastroenterology and Hepatology Diseases Research Center, Qom University of Medical Sciences, Qom, Iran; ${ }^{4}$ Department of Surgery, School of Medicine, Shahid Beheshti Hospital, Qom University of Medical Sciences, Qom, Iran; ${ }^{5}$ Department of Rheumatology, VU University Medical Center, Amsterdam, The Netherlands; ${ }^{6}$ Department of Health Information Technology, Tehran University of Medical Sciences, Tehran, Iran

Corresponding author: Sajjad Ahmadpour, Gastroenterology and Hepatology Diseases Research Center, Qom University of Medical Sciences, Qom, Iran

凶Email: sajjadahmadpour@yahoo.com

https://orcid.org/0000-0003-4321-874X

\section{References}

1. Zhu N, Zhang D, Wang W, Li X, Yang B, Song J, et al. A novel coronavirus from patients with pneumonia in China, 2019. N Engl J Med 2020; 382:727-33.

2. Dong Y, Mo X, Hu Y, Qi X, Jiang F, Jiang Z, et al. Epidemiological characteristics of 2143 pediatric patients with 2019 coronavirus disease in China. Pediatrics 2020;145:e20200702.

3. Heydari H, Eshagh Hossaini SK, Hormati A, Afifian M, Ahmadpour S. Coronavirus disease 2019 in a 2-month-old male infant: a case report from Iran. Clin Exp Pediatr 2020;63:499-502.

4. Taub JW, Ge Y, Xavier AC. COVID-19 and childhood acute lymphoblastic leukemia. Pediatr Blood Cancer 2020;67:e28400.

5. Alhuraiji A, Eldadah S, Alfraih F, Pandita R, Absi A, Hanbali A, et al. Optimal management of acute lymphoblastic leukemia (ALL) in adult patients during the novel coronavirus disease 2019 (COVID-19) pandemic. Gulf J Oncol 2020;1:7-18.

6. Chen Z, Xiong H, Li J, Li H, Tao F, Yang Y, et al. COVID-19 with postchemotherapy agranulocytosis in childhood acute leukemia: a case report. Zhonghua Xue Ye Xue Za Zhi 2020;41:341-3.

7. Yu J, Ouyang W, Chua ML, Xie C. SARS-CoV-2 transmission in patients with cancer at a tertiary care hospital in Wuhan, China. JAMA Oncol 
2020;6:1108-10

8. Liang W, Guan W, Chen R, Wang W, Li J, Xu K, et al. Cancer patients in SARS-CoV-2 infection: a nationwide analysis in China. Lancet Oncol 2020;21:335-7.

9. Pawar MSM. Multi system inflammatory syndrome in children and adolescents temporally related to COVID-19. Int J Multidiscip Res 2020; 1:97-102.

10. Kabeerdoss J, Pilania RK, Karkhele R, Kumar TS, Danda D, Singh S. Severe COVID-19, multisystem inflammatory syndrome in children, and Kawasaki disease: immunological mechanisms, clinical manifestations and management. Rheumatol Int 2021;41:19-32.
How to cite this article: Eshagh-Hosaini SK, Movahedi Z, Hormati A, Heydari H, Eshagh Hoseini SJ, Khodadust F, et al. Coronavirus disease 2019 in a 13-year-old patient with acute lymphoblastic leukemia. Clin Exp Pediatr 2021;64:247-50. https://doi.org/10.3345/cep.2020.01711 